\title{
Bearing Fault Diagnosis Using Motor Current Signature Analysis and the Artificial Neural Network
}

\author{
Thoalfaqqar Ali Dhomad ${ }^{\# 1}$, Alaa Abdulhady Jaber ${ }^{\# 2}$ \\ \# Mechanical Engineering Department, University of Technology, Baghdad, Iraq \\ E-mail: ${ }^{1}$ takaali17@gmail.com; ${ }^{2} 20039 @$ uotechnology.edu.iq
}

\begin{abstract}
Bearings are critical components in rotating machinery. The need for easy and effective bearings fault diagnosis techniques has led to developing different monitoring approaches. In this research, however, a fault diagnosis system for bearings is developed based on the motor current signature analysis (MCSA) technique. Firstly, a test rig was built, and then different bearing faults were simulated and investigated in the test rig. Three current sensors, type SCT013, were interfaced to Arduino MEGA 2560 microcontroller and utilized together for data acquisition. The time-domain signals analysis technique was utilized to extract some characteristic features that are related to the simulated faults. It was noticed that the simulated bearing faults have led to generating vibrations in the induction motors, which in turn cause a change in its magnetic field. For classification (identification) of the extracted features, the artificial neural network (ANN) was employed. An ANN model was developed using the Matlab ANN toolbox to detect the simulated faults and give an indication about the machine health state. The obtained features from the captured motor current signals were utilized for training the ANN model. The results showed the effectiveness of using MCSA based on the timedomain signal analysis in combination with ANN in diagnosis different bearings faults.
\end{abstract}

Keywords — bearing fault; health monitoring; MCSA; fault detection; time-domain signal analysis.

\section{INTRODUCTION}

Bearings are a very critical part of machines; however, they are cheap, but it represents the primary source of failure in the mechanical system. Faults in bearing may affect the other components in the machine, making them getting faulty, which leads to an increase in the maintenance cost. Faults in electrical motors bearing account for more than $40 \%$ of the induction motor failures [1]. Additionally, the gearbox's bearing failure is the top contributor to the wind turbines downtime. Thus, the early detection of the bearing fault is a significant concern in the industry.

Nevertheless, if the bearing fault is detected before getting severe, the maintenance time and the machine powering off can be correctly scheduled in a way that could not affect the system operation. That will also help the maintenance engineers to prepare the required spare parts before a while. Artificial neural networks (ANN) have widely being used for different applications, such as for human disease detection, mechanical fatigue life estimation, and electronics uses [2-4]. However, many studies have previously been carried out for the detection and diagnosis of different bearing faults based on various analysis techniques. For example, in 2009, Zarei, et al. [5] proposed an intelligent monitoring approach for bearing health assessment. Here, an intelligent filter has been designed using the ANN to remove the components that are not related to the bearing faults from the captured vibration signals. Then, the time-domain signal analysis was conducted on the filtered signals to extract features such as kurtosis, skewness, and root means square from them. The got features were fed into a second ANN designed for pattern recognition. Another research has dealt with motor faults detection when it is running at different loading conditions [6]. The detected faults include bearing and broken rotor bar faults. MCSA technique was effectively used along with the fuzzy logic method for fault classification.

In terms of the used advanced signal processing techniques in health monitoring Mehrjou, et al. [7] used the wavelet packet transform (WPT) to extracted essential features from the captured current signals. The detected faults were the static eccentricity and broken rotor bar in a three-phase squirrel cage induction motor. By using the WPT, the current signals were analyzed to multi-level subsignals; each contains a specific range of frequencies. Then, the FFT analysis and statistical features extraction, such as RMS and kurtosis, were carried out. Moreover, Widodo, et al. [8] have used the principle and independent component analysis for transient current signal analysis to extract its salient features. The support vector machines (SVM) technique was utilized for feature classification to diagnose 
induction motor faults. Pre-processing of the current signal was firstly conducted using the discrete wavelet transform. The researchers have mange to obtain 15 most significant features out of 63 extracted ones and thus reduced the required computation efforts. The developed intelligent system has experimentally validated and it was concluded that it can effectively be used for real machinery health monitoring applications.

Motor Current Signature Analysis (MCSA) is a condition monitoring technique, commonly used to diagnose the faults in mechanical systems. Its concept originates from the early 1970 s, and it was first proposed for condition monitoring in nuclear power plants for inaccessible mechanical equipment that is placed in hazardous areas [9]. Condition monitoring based on this technique is performed online without interrupting the production line while the motor is running under load at different operation conditions. MCSA can be used as a predictive maintenance tool for detecting the common faults in rotating machinery at its early stage and as such, prevent expensive catastrophic failures. Thus, it is going to be implemented in this research.

Reviewed previous studies have shown that the MCSA is a powerful technique in detection bearings faults without the need for direct attachment of sensors to the monitored bearing. Also, several methods can be used to analyze the motor current signals, such as time- and frequency-domain signal analysis techniques. Many features can be extracted after the signal being analyzed. These include, for instance, root mean square, kurtoses, skewness, and standard deviation and crest factor. The reviewed papers have shown that these features can give a clear indication about the bearing health situation if one (or more) of the artificial intelligence techniques are utilized. Thus, based on what has been reviewed, time-domain feature extraction along with ANN is going to be employed for bearing health monitoring. It aims to develop an intelligent, less complex, and easy to implement bearings condition monitoring system.

\section{MATERIALS AND METHOD}

This section gives a clear indication of the conducted experimental work, considering the test rig design, fault simulation, data acquisition system, and feature extraction. It describes all the performed experimental investigation on the current motor signature due to localized inner and outer race defects.

\section{A. Test Rig Design and Development}

The developed experimental test rig consists of threephase, 1 H.P. $(0.75 \mathrm{~kW}) \mathrm{AC}$ motor. It is connected via flexible couplings with a mild steel shaft of $2 \mathrm{~cm}$ diameter and $80 \mathrm{~cm}$ long.

TABLE I

SPECIFICATION OF 6304D BALL BEARING

\begin{tabular}{|c|c|}
\hline Number of balls $(\mathbf{N b})$ & 7 \\
\hline Max speed & $14000(\mathrm{rpm})$ \\
\hline Bore diameter & $20(\mathrm{~mm})$ \\
\hline Outside diameter & $52(\mathrm{~mm})$ \\
\hline The contact angle of bearing & 0 \\
\hline
\end{tabular}

The shaft is mounted on two identical deep groove ball bearings, which are fixed in two pillow blocks attached with a cast-iron frame. The specification of the utilized bearings is shown in Table I.

The induction motor was connected to a variable frequency drive (VFD) N700E inverter from Hyundai to control the rotation speed. The frame was filled with sands to give the test rig more operation stability. The load was arranged to be applied in the middle of the two bearings via carrying the weight using a simple mechanism supported on a third neglected bearing, as shown in Fig. 1.

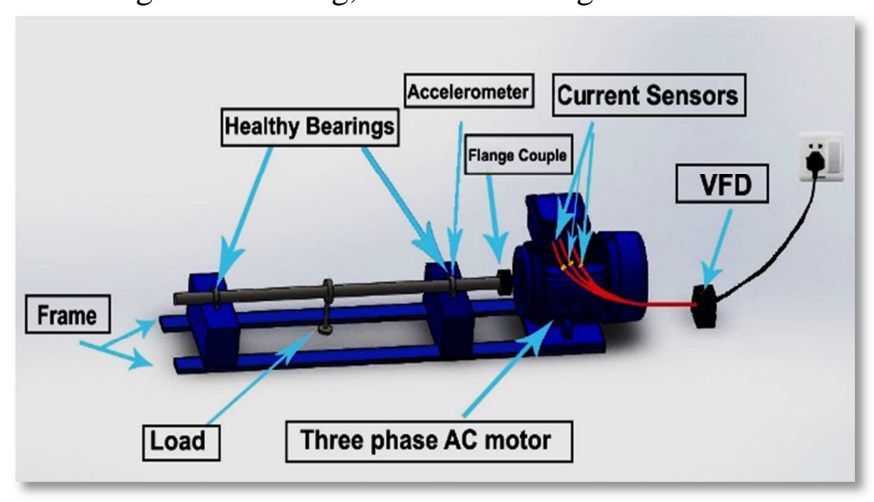

Fig. 1. The designed test rig

\section{B. Data Acquisition System}

The data acquisition system (DAS) is a system composed of hardware and software. It is used to interface the test rig to a PC in order to measure and monitor different variables, such as temperature and vibration. The developed DAS in this research is discussed as follows [10].

The hardware components of the acquisition system are carefully selected and used to monitor and record the current reading of the three-phase induction motor when different applied load and different rotating speed at several bearing faults. It is consisted of three hall effect current sensors (SCT013), as shown in Fig. 2, with a maximum range of 30 $\mathrm{A}$ and $5 \mathrm{~V}$ input.

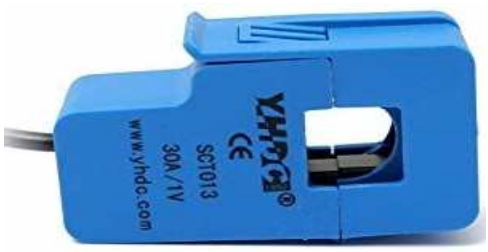

Fig. 2 SCT013 Sensor

These sensors were calibrated using a clamp meter. The three current sensors were connected to Arduino MEGA 2560 (Fig. 3).

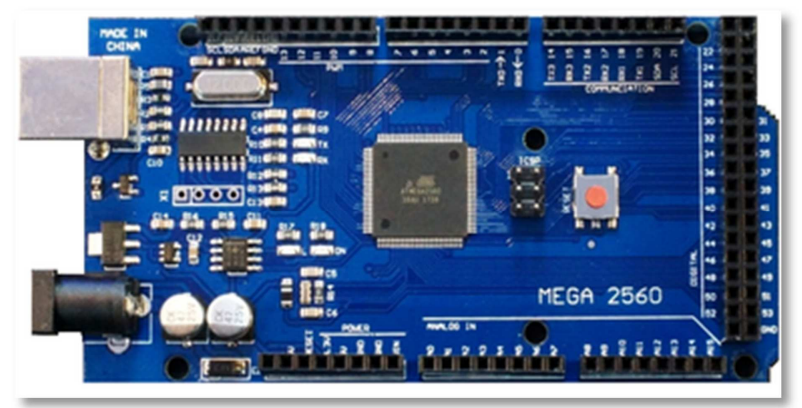

Fig. 3 Arduino MEGA 2560 
Here, the utilized Arduino microcontroller has 54 input/output pins (of which 15 can be used as PWM outputs), 16 analog inputs, 4 UARTs (hardware serial ports), a 16 $\mathrm{MHz}$ crystal oscillator, a USB connection, a power jack, an ICSP header, and a reset button. However, the microcontroller and the sensors were chosen carefully according to their sensitivity, cost, and availability.

The software part of the data acquisition system is mainly based on the Arduino Integrated Development Environment (IDE). The Arduino microcontroller is programmed to read the signals from the three current sensors and print them on the serial port with a $100 \mathrm{~Hz}$ sampling rate to capture 4000 samples, as shown in Fig. 4.

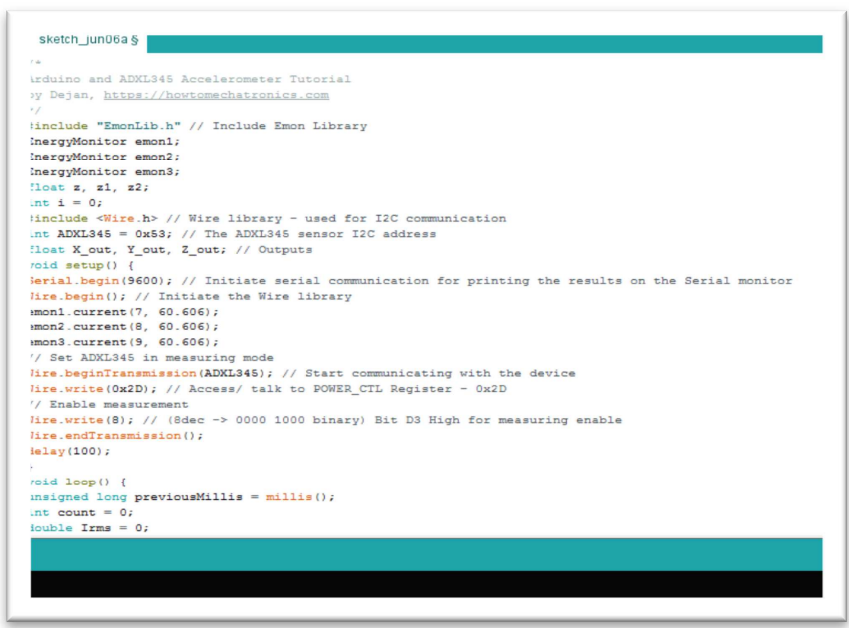

Fig. 4 The developed Arduino code

\section{Faults Simulation}

The most common bearings faults are inner race faults, outer race faults, and cage faults. In this study, $3 \mathrm{~mm}$ inner race and $3 \mathrm{~mm}$ outer race defects are simulated, as shown in Fig. 5.

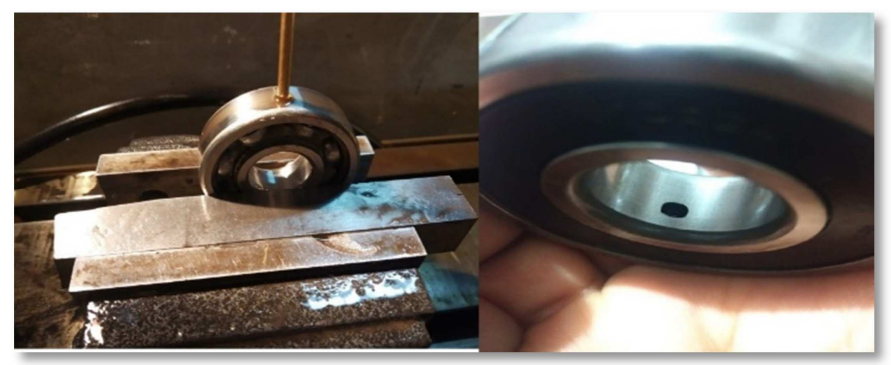

Fig. 5 Inner race and outer race faults simulation

These faults were made using the electrical discharge machining (EDM) technique. The utilized machine is of type CM323C spark machine; a rod of copper with a $3 \mathrm{~mm}$ diameter was used to make circular holes on the inner and outer races. The machine was set to $0.4 \mathrm{~mm}$ as the feed rate with a floating voltage ranging from 0 to $100 \mathrm{~V}$. To study the effect of these faults using the MCSA, the results from 15 experiments conducted on the developed test rig, considering different rotating speeds, were investigated. The considered test rig operation conditions that are followed to study the effect of the fault are presented in Table II.
TABLE II

SETS OF THE CONDUCTED EXPERIMENTAL SETUP

\begin{tabular}{|c|c|c|}
\hline Bearing health state & $\begin{array}{c}\text { Loading } \\
\text { condition }\end{array}$ & $\begin{array}{c}\text { Motor } \\
\text { frequency }\end{array}$ \\
\hline Healthy & Free weight & $15 \mathrm{~Hz}$ \\
\hline Healthy & Free weight & $25 \mathrm{~Hz}$ \\
\hline Healthy & $1 \mathrm{~kg}$ & $25 \mathrm{~Hz}$ \\
\hline Healthy & $1.5 \mathrm{~kg}$ & $25 \mathrm{~Hz}$ \\
\hline Healthy & $2.5 \mathrm{~kg}$ & $25 \mathrm{~Hz}$ \\
\hline Inner race fault & Free weight & $15 \mathrm{~Hz}$ \\
\hline Inner race fault & Free weight & $25 \mathrm{~Hz}$ \\
\hline Inner race fault & $1 \mathrm{~kg}$ & $25 \mathrm{~Hz}$ \\
\hline Inner race fault & $1.5 \mathrm{~kg}$ & $25 \mathrm{~Hz}$ \\
\hline Inner race fault & $2.5 \mathrm{~kg}$ & $25 \mathrm{~Hz}$ \\
\hline outer race fault & Free weight & $15 \mathrm{~Hz}$ \\
\hline outer race fault & Free weight & $25 \mathrm{~Hz}$ \\
\hline outer race fault & $1 \mathrm{~kg}$ & $25 \mathrm{~Hz}$ \\
\hline outer race fault & $1.5 \mathrm{~kg}$ & $25 \mathrm{~Hz}$ \\
\hline outer race fault & $25 \mathrm{~kg}$ & $25 \mathrm{~Hz}$ \\
\hline
\end{tabular}

\section{Time-Domain Signal Analysis for Feature Extraction}

Signals are carrying the vital information and features of the monitored system. The extracted features from the signal can be classified as stationary or non-stationary. Based on the nature of the features, the stationary feature can also be classified into deterministic and nondeterministic. Deterministic signals have specific frequency components and can be described by a mathematical equation [11]. Timedomain features are going to be used here as the input to the ANN, and they can be extracted from the captured signals using the following relationships [12]:

1) Root mean square (RMS): measures for all levels of a signal. Mathematically it is expressed as:

$$
R M S=\sqrt{\frac{1}{N} \sum_{i=1}^{i=N} X_{i}^{2}}
$$

2) Standard Deviation (std): This parameter measures the desperation of the signal about the mean and mathematically can be expressed as:

$$
S T D=\sqrt{\frac{1}{N} \sum_{i=1}^{i=N}\left(X_{i}-\bar{X}\right)^{2}}
$$

3) The Crest Factor $(C F)$ : it is the ratio of maximum peak to the RMS value, and it indicates the impact occurred in the signal [13]. It can be computed as in the following:

$$
C F=\frac{\max \left|X_{i}\right|}{R M S}
$$

4) Kurtosis (Ku): The fourth moment, normalized concerning the fourth power of standard deviation, is quite useful in fault diagnosis. This value is called kurtosis, which is a compromise measure between the intense lower moments and other sensitive higher moments. It is calculated according to the following equation: 


$$
K u=\frac{\sum_{i=1}^{i=N}\left(X_{i}-\bar{X}\right)^{4}}{(N-1) *(s t d)^{4}}
$$

5) Skewness (Sk): it measures the symmetry level of vibration signals through its probability density function (PDF) [13]. Equation (5) defines the third moment or skewness as:

$$
S k=\frac{\sum_{i=1}^{i=N}\left(X_{i}-\bar{X}\right)^{3}}{(N-1) *(s t d)^{3}}
$$

For normally distributed data sets, the odd moments are zero unless the time domain signal is rectified. Hence, skew can easily track the bearing health condition [13]. However, $X_{i}$ is a vector of the signal samples is, $\bar{X}$ is the mean of the collected samples, and $N$ is the number of samples, respectively.

\section{E. The Artificial Neural Network}

The artificial neural networks (ANN) are generally based on the mathematical processing of data, and it is frequently utilized for fault detection and diagnosis. The ANN is mainly divided into two types: supervised and unsupervised. The difference between them is based on the applied method in training the network. In supervised learning, a "teacher" is presented during the learning process, where the ANN has a given input (target), and the error is the difference between ANN output and the target. The ANN weights are then adjusted using the back-propagation algorithm to reduce the difference between the actual and the desired outputs. In this way, the ANN is trained to learn a specific behavior and outputs the desired result for each input based on the presence of training patterns, which makes this learning is a supervised type [14]. However, with unsupervised learning, there is no target available to the ANN, and only the input is presented. In this type of ANN, the learning approach is achieved by discovering and adapting the structure of the features in the input patterns. This is conducted by adapting statistical regularities or clustering patterns in the training samples. Here the network organizes itself to develop properties of the training data set that emerge as the training process proceeds [15].

The structural unit of ANN is the neuron, which consists of summer and an activation function, as shown in Fig. 6. Where $X_{1}, X_{2}, X_{3}, \ldots, X_{n}$ are the inputs to the neuron with corresponding weights $\mathrm{W}_{1}, \mathrm{~W}_{2}, \mathrm{~W}_{3} \ldots, \mathrm{W}_{\mathrm{n}}$, which model the synaptic neural connections in biological nets and act in such a way as to increase or decrease the input signals to the neuron. Sometimes a threshold term $(b)$ could be added to the inputs. Generally, inputs, weights, thresholds, and neuron output could be the real value or binary or bipolar. All inputs are multiplied by their corresponding weights and added together to form the net input to the neuron. The mathematical expression for the net can be written as in Equation (6) [16]. The neuron behaves as an activation or mapping function $f(n e t)$ to produce an output $Y$, which can be expressed as in Equation (6).

$$
\begin{gathered}
\text { Net }=\sum_{i=1}^{i=n} W_{i} X_{i}+b=W_{1} X_{1}+W_{2} X_{2}+W_{3} X_{3}+ \\
\cdots . W_{n} X_{n}+b \quad \\
Y=f\left(\sum_{i=1}^{i=n} W_{i} X_{i}+b\right)
\end{gathered}
$$

Where $f$ is called the neuron activation function or the neuron transfer function. The standard neuron activation functions are linear, threshold, log-sigmoid, and tan-sigmoid. All these functions are squashing since they limit the neuron output to asymptotic levels. Using nonlinear activation functions allows nonlinear input-output mapping of $\mathrm{NN}$, which can permit nonlinear function approximation [17].

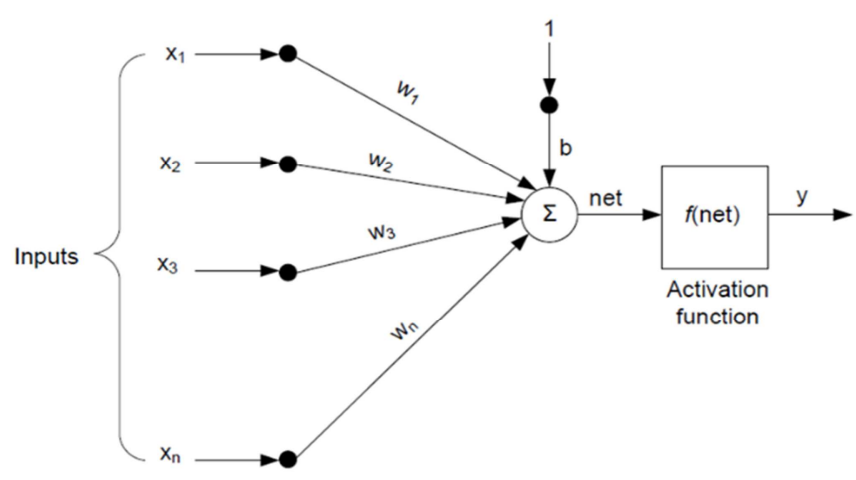

Fig. 6 Structure of Artificial Neuron

\section{F. ANN Design}

In this research, the neural network toolbox under Matlab $\mathrm{R} 2016 \mathrm{~b}$ has been used to determine the neural network structure. However, some parameters need to be carefully considered when designing any ANN model. These include hidden layers number, neurons in each hidden layer, and type of activation factions. Also, the number of training iterations and the learning rate value must be cautiously tuned. Several models have been tested based on changing the number of hidden layers and their neurons, with different types of activation functions and training algorithms. The input data to the ANN model were the extracted timedomain features, while three output parameters from the ANN model are realized to identify the bearing health condition. The obtained best number of hidden layers is one layer with 46 neurons, and tan-sigmoid activation function (Fig. 7).

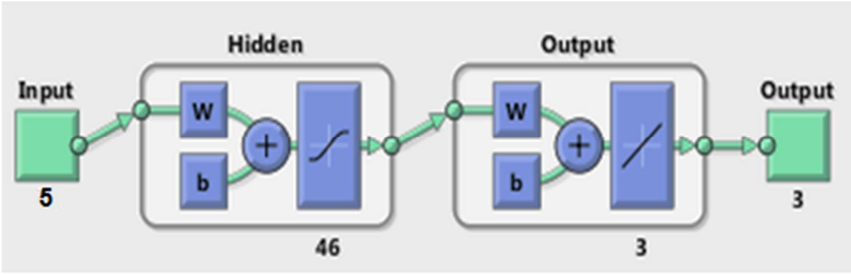

Fig. 7 The obtained best ANN design

Depending on the trial and error approach, all the training techniques have been tested and compared in terms of the mean square error (MSE) and correlation factor. From the results, it was established that the Bayesian regularization backpropagation (trainbr) algorithm has less MSE with the best correlation coefficient, which were 0093 and 0.98 , respectively, so it was chosen as the best training algorithm in this research.

\section{RESULT AND DISCUSSION}

The occurrence of a fault in the mechanical machines is leading to alter the stiffness around the fault. Electrical 
motors, however, produce noise and vibration, because of the internal magnetic, mechanical and aerodynamic forces. Thus, this can generate an impulse or shock, which leads to further consequence in the variation of the collected vibration or electrical signals. MCSA represents an effective technique that can be used for load variation and fault detection [9]. However, in this study, three different states of bearing health (healthy, inner race and outer race faults) have been considered. Motor current signal capturing was conducted utilizing Arduino MEGA 2560. Nevertheless, before data capturing when bearing fault was presented, the test rig was run for a short time to permit the rotating system to settle dynamically. Then, different time domain features were calculated based on the theoretical equations that were presented previously. In the experimental work, the developed test rig was run at different operation/health conditions (shown in Table II). Four thousand samples of the current signal were taken at each experiment; these significant signals are divided into sub-signals (using MS Excel) with 100 samples each.

\section{A. Captured Current Signals}

To investigate the effect of loading conditions on the current signal when the bearing is healthy and faulty (inner and outer race faults), the test rig was operated at a fixed rotating frequency while the applied load is increased gradually. The captured current signals are indicated in Fig. 8 below.

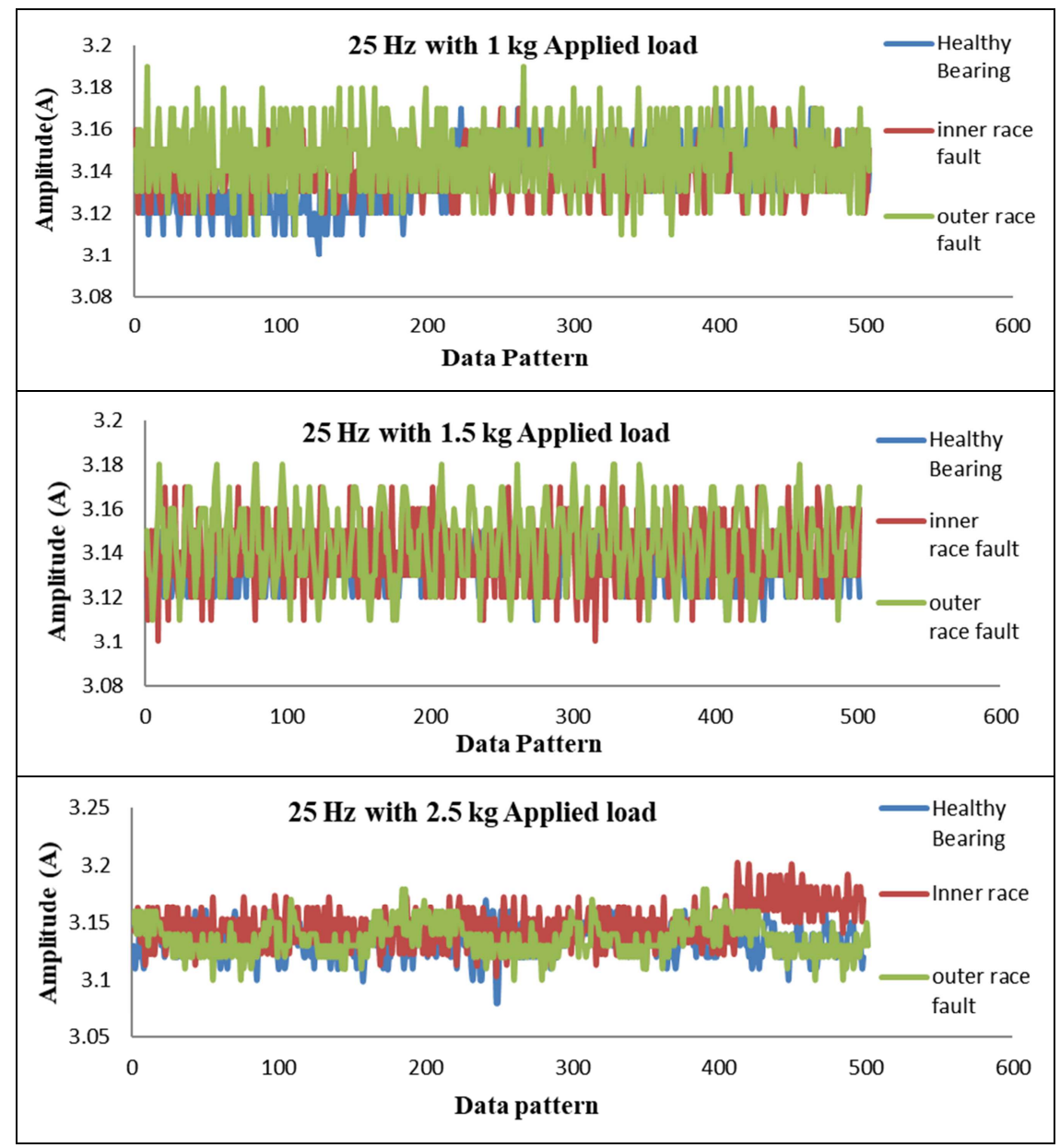

Fig. 8 Stator current signal at the three bearing health states with $25 \mathrm{~Hz}$ and different applied loads

It can be noticed that the amplitude of the current spectrum gets higher with the increase of the applied load. However, the signal amplitude has a much higher change with respect to change in the applied load when the outer race fault is introduced. This could be due to the impact that occurs between the rolling element and the simulated fault that produces an impulse and excites the resonance frequencies of the rotating system. Moreover, this can also be related to the outer race of the bearing that always carries out most of the applied load than the inner race. From this, it can be concluded that the outer race defect is more dangerous, more sensitive to the applied load, and has more effect on the overall vibration level of the rotating system.

\section{B. Features Extraction}

To further investigate varying the loading effect and also to calculate sets of statistical values from the time-domain signals, which are used in developing the ANN, the root mean square (RMS), standard deviation (STD), crest factor $(\mathrm{CF})$, kurtosis (KU), and skewness (SK) values for each loading condition at different bearing health state were extracted and compared, as illustrated in the following figures. Figure (9) to Figure (13) shows the values of RMS, STD, CF, KU, and SK for the captured current signal after being divided into sub-signals. Where the $\mathrm{X}$-axis represents the number of data sets (data pattern), and Y-axis is the value of the calculated feature. However, an important 
observation that can be noticed from these figures is that the computed time-domain (statistical) features are altered as the fluctuation occurs in the captured random current signals. Generally, from the figures mentioned above can be noticed that the values of RMS, STD, CF are higher when outer race fault is simulated, less in the case of inner race fault, and typically lesser when the bearing is healthy. This is the same for all the three loading cases. Also, these features are clearly separated based on the simulated health state. The existing impulse in the signals can also be measured using kurtosis and skewness features. These two parameters are suitable in detecting the incipient faults and in the detection of any change that could happen in the operating conditions. Kurtosis and skewness have high sensitivity to the early faults, and as the severity degree increases, they increase gradually.

However, for more severe faults, they could unexpectedly decrease. This has obviously been noticed in this study, where the kurtosis value is less for the faulty health conditions, as shown in Figure (12). The trends of kurtosis values are got decreased with the outer and inner race faults and then got increased in the healthy state (Figure 12). Therefore, it can be concluded that the kurtosis value gets unsuitable to the faults with high severity. In terms of skewness values that are shown in Figure (13), it can be observed that its trends for the three bearing health states are overlapped with each other and cannot extract any useful information from them. Generally, it can be stated that the time domain statistical features can reflect the mechanical health conditions from different points of view, as they can recompense each other.

On the other hand, it is diverse for each indicator to comprise fault information in different fault harshness levels. Even though, to some extent, many features cannot imitate the changes of faults. Therefore, it is advised that more features should be computed to diagnose the faults of rotating machinery successfully. However, in this research, the previously mentioned features (RMS, STD, CF, KU, and SK) are computed and used since the proposed use of the ANN is expected to assess the suggested fault diagnosis approach in this study effectively.

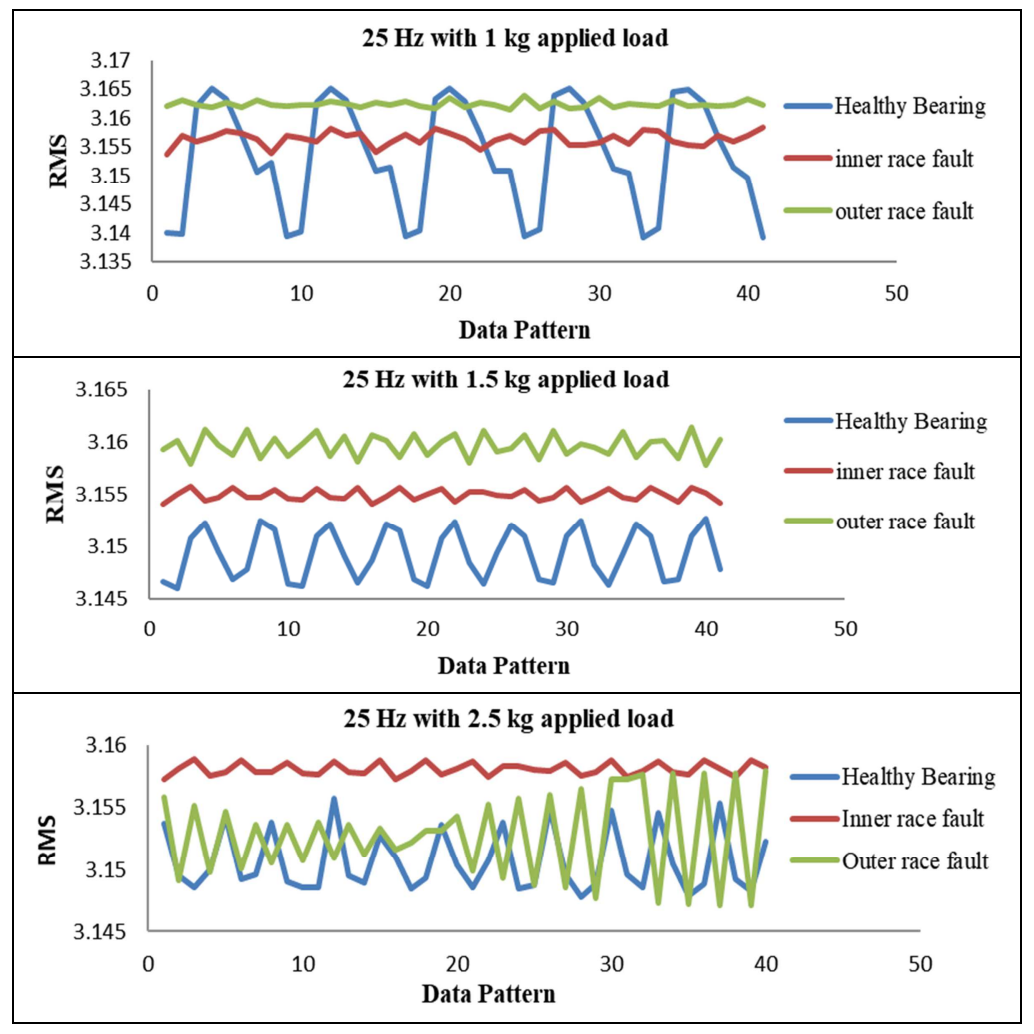

Fig. 9 : Variation of RMS with the Applied load

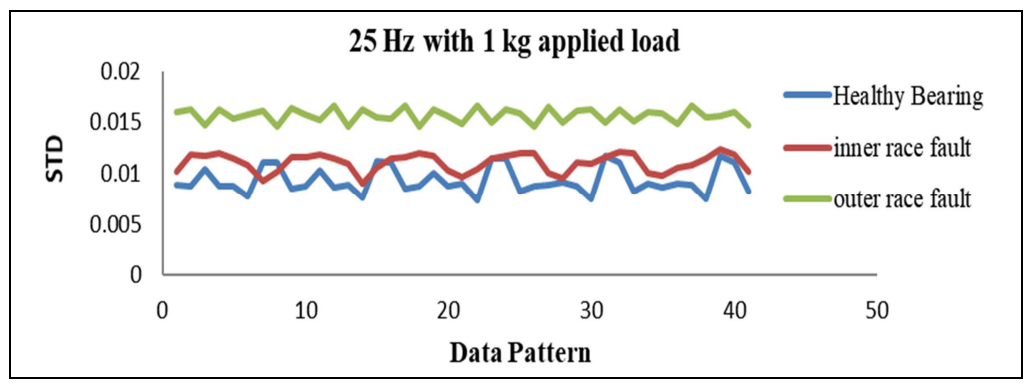




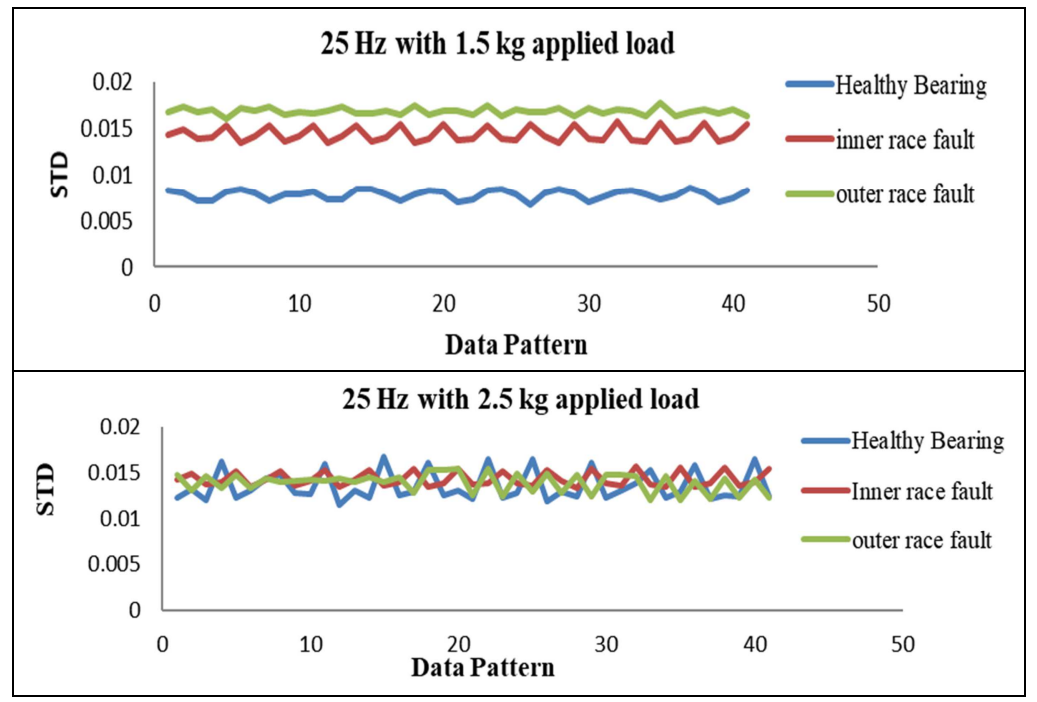

Fig. 10 Variation of STD with Applied Load
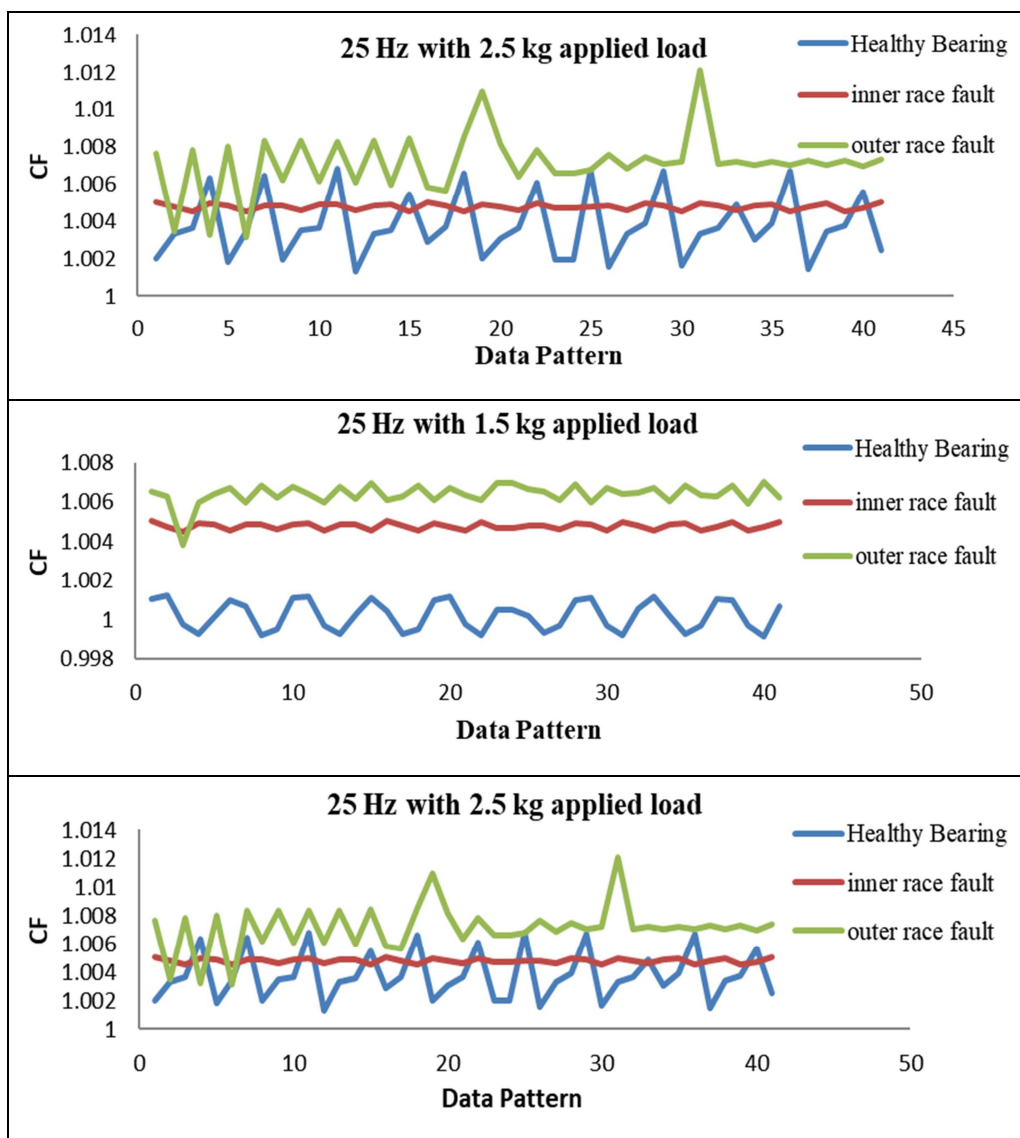

Fig.11 Variation of CF with Applied Load

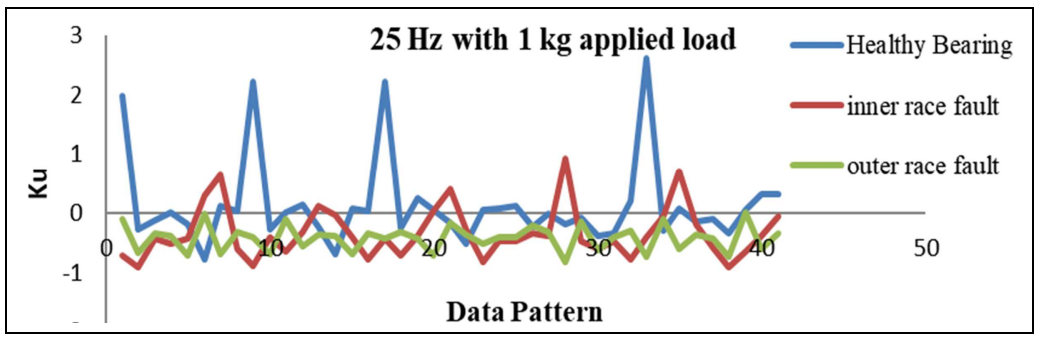




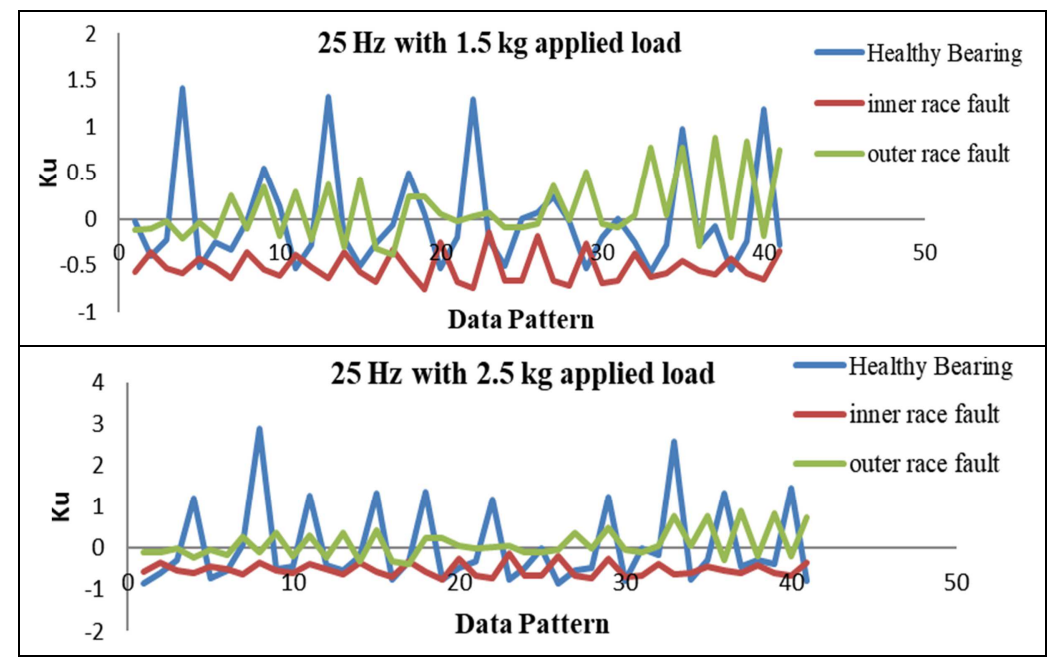

Fig. 12 Variation of Kurtosis with Applied Load

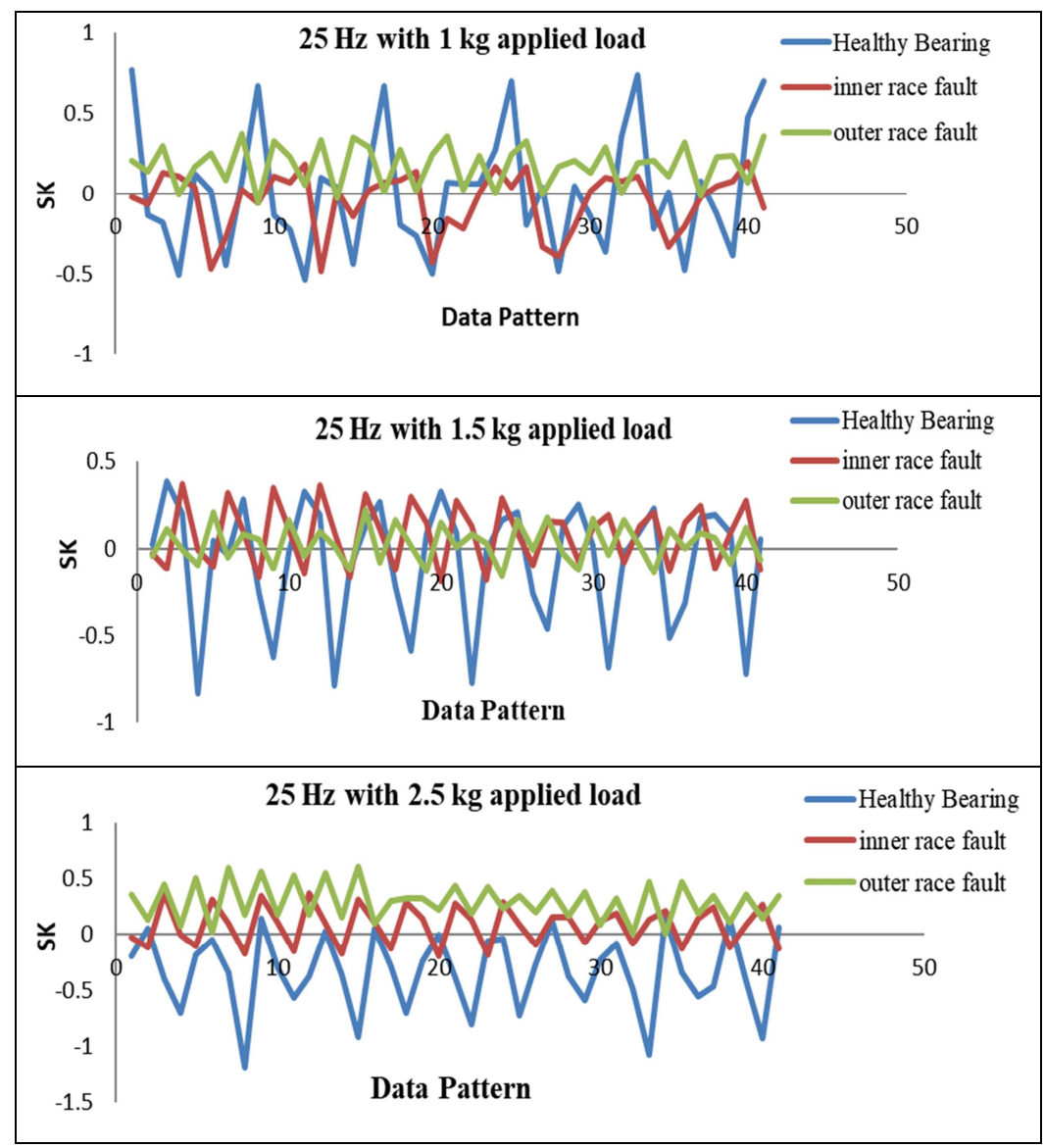

Fig. 13 Variation of Skewness with Applied Load

\section{Testing and validation}

The validation test of the developed ANN was done by using unseen data, not previously used in designing the ANN model, consist of 360-sets; every 120 sets represent a bearing health condition. However, only one fault type is presented at a time. A binary classification approach was adopted to identify the bearing health conditions. For instance, if the output of the ANN model is $\left(\begin{array}{lll}1 & 0 & 0\end{array}\right)$, the bearing is healthy while there is an inner or outer race fault if the output is $\left(\begin{array}{lll}0 & 1 & 0\end{array}\right)$ or $\left(\begin{array}{lll}0 & 0 & 1\end{array}\right)$, respectively. Figure (14) shows the ANN classification results where it can be noticed that when the bearing was healthy, the first neuron in the output layer is one and remains as it is for the first 120 data sets, whereas the other neurons are zeroes. Then, the second neuron becomes 1 when the inner race fault is introduced and the other neurons are zeroes and so on for outer race fault where the third neuron becomes 1, and the other neurons are zeros. From the result in Figure (14), it can be clearly seen that the faults are easily identified. Thus, the designed ANN is feasible for the application in this research. 


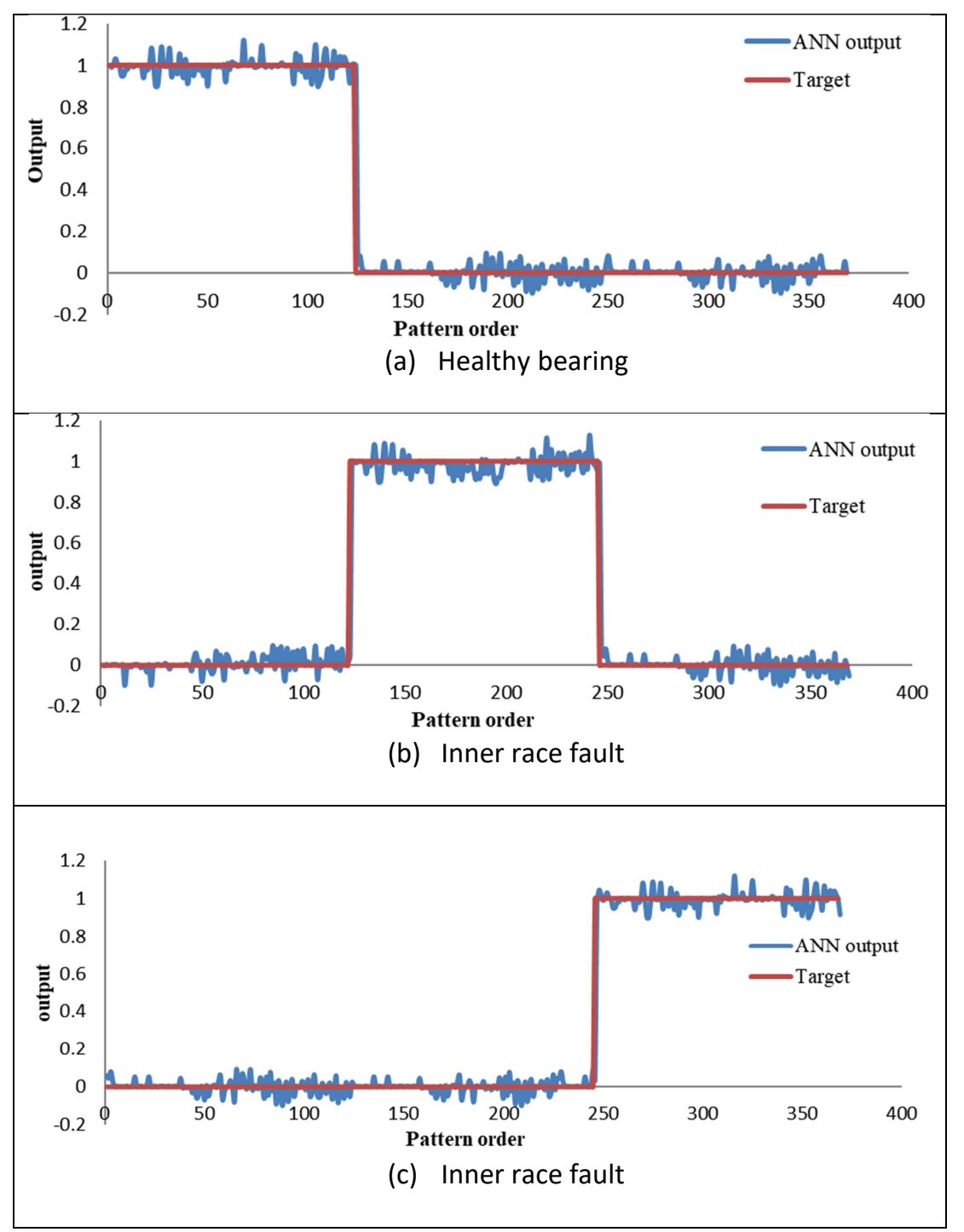

Fig. 14 Result of the designed ANN when unseen data are presented

\section{IV.CONCLUSION}

In this research, a fault diagnoses system for bearings has been developed based on feature extraction from motor current signals. The followed fault diagnosis approach in this research is commonly known as motor current signature analysis (MCSA). The extracted features were then classified using the artificial neural network. After accomplishing this research, the following conclusions can be drawn.

- MCSA can effectively detect the bearing faults by monitoring the current signals of the induction motor.

- The effect of bearing's fault can change concerning the motor speed and applied load.

- The outer race bearing's fault has a much higher effect with the applied load than the inner race fault; this could be because of the outer race is carrying most of the applied load than the inner race.

- The features extraction from current signals such as (RMS, kurtoses, etc.) can give a good indication about the bearing's state.

- Using the ANN for features classification represents a powerful technique to diagnose different types of bearings' faults by training it with the features that were extracted from current signals.

- Time-domain signal analysis can effectively be used for signals analysis to extract salient features related to the simulated faults. It also considered an easy to implement signal analysis technique.

- Utilizing Arduino microcontroller for data acquisition shows a reliable and cost-effective tool for building efficient fault diagnosis systems.

However, in this study, just one fault is detected at a time. Thus, it is recommended to consider simulation and detection of more than one fault at the same time. Also, the wireless capability could be added to the data acquisition system in order to monitor the system under investigation from far and different places. The wireless system could also be complemented with a mobile application in order to let the in charge personal to view the state of monitored machines at any wanted time.

\section{REFERENCES}

[1] B. Rao, Handbook of condition monitoring: Elsevier, 1996.

[2] A. O. Ibrahim, S. M. Shamsuddin, A. Y. Saleh, A. Ahmed, M. A. Ismail, and S. Kasim, "Backpropagation neural network based on 
local search strategy and enhanced multi-objective evolutionary algorithm for breast cancer diagnosis," International Journal on Advanced Science, Engineering and Information Technology, vol. 9, pp. 609-615, 2019.

[3] M. F. M. Yunoh, S. Abdullah, and S. S. K. Singh, "Artificial neural network classification for fatigue feature extraction parameters based on road surface response," International Journal on Advanced Science, Engineering and Information Technology, vol. 8, pp. 14801485, 2018.

[4] G. C. Cardarilli, L. Di Nunzio, R. Fazzolari, M. Re, and S. Spano, "AW-SOM, an Algorithm for High-speed Learning in Hardware Self-Organizing Maps," IEEE Transactions on Circuits and Systems II: Express Briefs, 2019.

[5] J. Zarei, M. A. Tajeddini, and H. R. Karimi, "Vibration analysis for bearing fault detection and classification using an intelligent filter," Mechatronics, vol. 24, pp. 151-157, 2014/03/01/ 2014.

[6] S. Shukla, M. K. Jha, and M. S. F. Qureshi, "Motor Current Signature Analysis for Fault Diagnosis and Condition Monitoring of Induction Motors using Interval Type-2 Fuzzy logic," ed, 2014.

[7] M. R. Mehrjou, N. Mariun, M. Karami, S. B. M. Noor, S. Zolfaghari, N. Misron, et al., "Wavelet-Based Analysis of MCSA for Fault Detection in Electrical Machine," in Wavelet Transform and Some of Its Real-World Applications, ed: IntechOpen, 2015.

[8] A. Widodo, B.-S. Yang, D.-S. Gu, and B.-K. Choi, "Intelligent fault diagnosis system of induction motor based on transient current signal," Mechatronics, vol. 19, pp. 680-689, 2009.
[9] S. Singh, A. Kumar, and N. Kumar, "Motor current signature analysis for bearing fault detection in mechanical systems," Procedia Materials Science, vol. 6, pp. 171-177, 2014.

[10] A. A. Abouhnik, "An Investigation into Vibration Based Techniques for Wind Turbine Blades Condition Monitoring," Manchester Metropolitan University, 2012.

[11] A. A. Jaber and K. M. Ali, "Artificial Neural Network Based Fault Diagnosis of a Pulley-Belt Rotating System," International Journal on Advanced Science, Engineering and Information Technology, vol. 9, pp. 544-551, 2019.

[12] J. K. Sinha, Vibration analysis, instruments, and signal processing: CRC press, 2014.

[13] W. Caesarendra and T. Tjahjowidodo, "A Review of Feature Extraction Methods in Vibration-Based Condition Monitoring and Its Application for Degradation Trend Estimation of Low-Speed Slew Bearing," Machines, vol. 5, p. 21, 2017.

[14] J. M. Zurada, Introduction to artificial neural systems vol. 8: West publishing company St. Paul, 1992.

[15] R. A. Ashnibha, "An investigation into current and vibration signatures of three phase induction motors," Manchester Metropolitan University, 2012.

[16] K. Mehrotra, C. K. Mohan, and S. Ranka, Elements of artificial neural networks: MIT Press, 1997.

[17] D. W. Patterson, Artificial neural networks: theory and applications: Prentice Hall PTR, 1998. 